\title{
Calcium carbonate-enriched cheese to improve nutrition, compliance and phosphorus control in patients on kidney replacement therapy
}

\author{
Gianluigi Ardissino ${ }^{1} \cdot$ Roberto Matta $^{1} \cdot$ Mariagrazia Patricelli $^{1} \cdot$ Valentina Capone ${ }^{1} \cdot$ Antonio Leoni $^{1}$. \\ Antonio Groppelli ${ }^{2}$. Simone Vettoretti ${ }^{3}$. Laura Pavone ${ }^{3}$. Elisabetta Margiotta ${ }^{3}$ - Annamaria Brancadoro ${ }^{3}$. \\ Emanuele Grimaldi ${ }^{3} \cdot$ Isabella Cropanese ${ }^{4} \cdot$ Francesca Raffiotta $^{3} \cdot$ Piergiorgio Messa $^{3} \cdot$ Giovanni Montini $^{1}$
}

Received: 18 March 2021 / Accepted: 18 June 2021 / Published online: 29 June 2021

(c) Italian Society of Nephrology 2021

\begin{abstract}
Background Patients on renal replacement therapy face many dietary limitations, and cheese is often limited because of its high phosphate content; we have developed cheese with added calcium carbonate $\left(\mathrm{CaCO}_{3}\right)$ to provide patients with a nutritional opportunity while improving their phosphate control.

Methods The present double-blind crossover study was aimed to compare the new modified cheese with an equivalent standard product in 16 patients. The increase in inter-dialysis phosphorus $(\Delta \mathrm{P})$ and pre-dialysis calcium were used as the primary endpoints for efficacy and safety.

Results The median $\Delta \mathrm{P}$ (and IQR) was significantly lower with the modified cheese compared with the standard product: 2.5 (1.9-2.9) mg/dL vs. $2.7(2.2-3.4) \mathrm{mg} / \mathrm{dL}$, respectively $(\mathrm{p}<0.02)$. No difference was observed in pre-dialysis serum calcium levels.

Conclusions The described modified cheese may represent an interesting means of overcoming some of the dietary limitations in patients on dialysis to help them achieve better nutrition and quality of life.

\section{Graphic abstract}

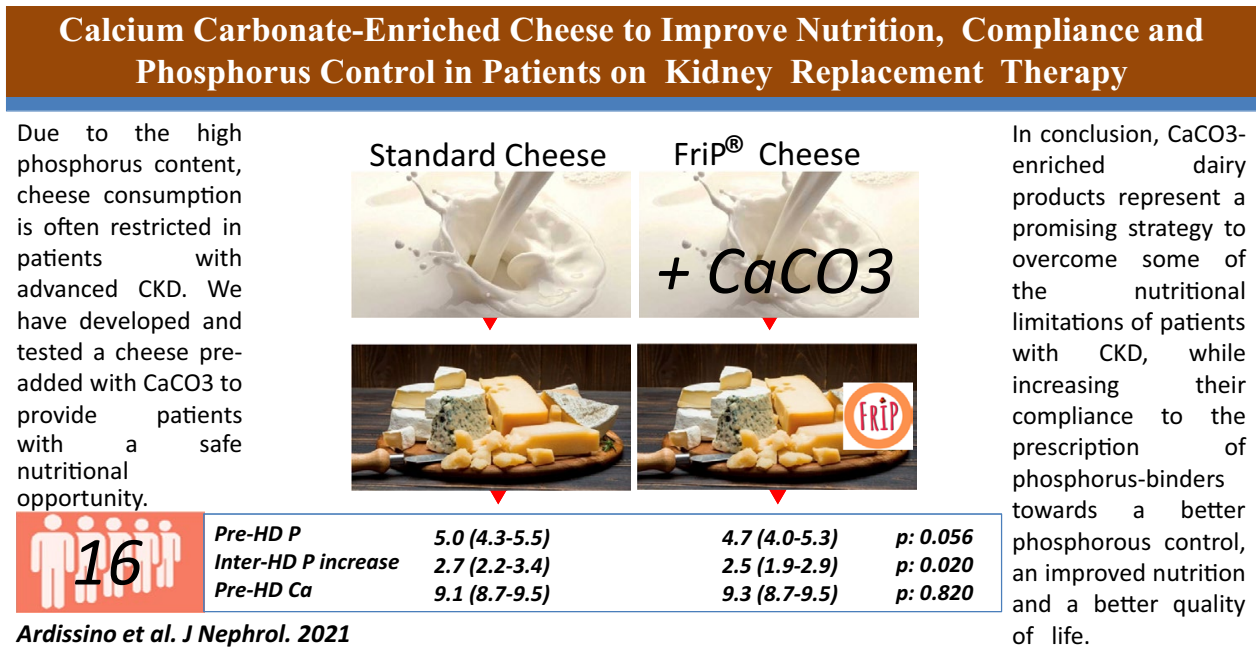

Keywords Phosphorus $\cdot$ Renal replacement therapy $\cdot$ Dialysis $\cdot$ Secondary hyperparathiroidism

Gianluigi Ardissino

ardissino@centroseu.org

Extended author information available on the last page of the article 


\section{Introduction}

Patients with advanced chronic kidney disease (CKD) have several dietary limitations that make their diet unappealing, leading to detrimental consequences, such as poor adherence to prescriptions, malnutrition and overall poor quality of life [1]. Cheese is an important component of the European diet but, due to its high phosphorus (as well as salt and animal fat) content, its consumption is often restricted in patients on kidney replacement therapy (KRT) [2]. To overcome some of the above-mentioned dietary limitations, we developed a special cheese with a fixed amount of calcium carbonate $(\mathrm{CaCO} 3)$ added to the milk during the production process (FriPß). The present study aimed to test this new product in patients on hemodialysis.

\section{Methods}

In a pilot study, we tested the efficacy of the daily consumption of a special cheese produced from cow's milk supplemented with a fixed amount of $\mathrm{CaCO}_{3}$ on serum phosphorus levels in a sample of patients undergoing chronic hemodialysis three times per week. The secondary end points were serum calcium levels, palatability and average consumption of FriP cheese in our population. FriP cheese was produced according to the best cheesemaking tradition so as to be indistinguishable from standard cheese. After a run-in period of 1 month on their usual diet, patients were randomly assigned to receive either the standard cheese followed by the modified cheese (FriP) for 1 month each, or the opposite sequence in a doubleblind fashion. A washout period of 1 week was introduced between treatment periods.

The composition per $100 \mathrm{gr}$ of product of both cheeses was the same: calories $369 \mathrm{kcal}$; Fat $25.8 \mathrm{gr}$ (of which $17.6 \mathrm{gr}$ is saturated fat); carbohydrates $<0.3 \mathrm{gr}$; protein $20.0 \mathrm{gr}$; sodium $1 \mathrm{gr}$ and phosphorus $591 \mathrm{mg}$. The only difference was the calcium content which was $966 \mathrm{mg}$ in the standard cheese and $1238 \mathrm{mg}$ in FriP cheese. During the study, the dialysis prescription and the use of both phosphorus-binders and vitamin D therapy were not modified. The end points were the increase in inter-dialysis $(48 \mathrm{~h})$ serum phosphorus $(\Delta \mathrm{P})$, predialysis phosphorus levels and predialysis calcium levels, which were regularly and repeatedly measured during each of the study periods (at least 5 measurements per period). The additional parameters measured at the end of each study period (predialysis) were body weight, blood pressure, urea, creatinine, total cholesterol, triglycerides, albumin, alkaline phosphatase, parathyroid hormone (PTH), ionized calcium and bicarbonate. Patients were instructed to eat the provided cheese during or after their main meals no more than once daily. Throughout the study period, patients were provided with $500 \mathrm{gr}$ of cheese (as appropriate according to randomization) and the dietitian (RM) regularly asked how much of the previously provided product had been consumed (none/one-fourth/half/three-fourths/all) and how much they enjoyed it (not much/average/very much).

Statistical analysis: Categorical variables are given in absolute numbers and discrete variables as median and interquartile range (IQR). Statistical analysis was tested with the Mann-Whitney test. Chi-square test was used to compare categorical variables. Statistical significance was set at a $p$ value of $<0.05$ (2-tailed). Data analysis was performed using StatView (Abacus Corp., California, USA).

\section{Results}

Twenty-two patients were enrolled, 16 of whom successfully completed the 2 treatment periods. There were 8 teenagers ( 4 females) and 8 adults ( 6 females). All patients were Caucasian, had been on extracorporeal dialysis for at least 6 months and were on a thrice weekly regimen. Drop-outs were due to transplantation (n: 3 ), reported non-adherence to the protocol (n: 2) or to SARS-CoV-2 infection (n: 1). Table 1 describes the general characteristics of the studied patients and the laboratory parameters at baseline and at the end of each study period.

The observed medians of the interdialysis $\Delta \mathrm{P}$ (and the IQR) were as follows (Fig. 1): run-in: $2.7(2.4-3.5) \mathrm{mg} /$ dL; standard cheese: $2.7(2.2-3.4) \mathrm{mg} / \mathrm{dL}$; and FriP cheese $2.5(1.9-2.9) \mathrm{mg} / \mathrm{dL}$ ( $\mathrm{p}<0.02$ for FriP vs. both run-in and standard cheese). Individual responses regarding efficacy (inter-dialysis $\Delta \mathrm{P}$ ) are provided in Fig. 2 with all but 3 patients experiencing a decrease in $\Delta \mathrm{P}$ during the period in which they were consuming the FriP cheese compared to the period in which they were consuming the standard cheese. Predialysis phosphorus levels were also lower while consuming FriP cheese than while consuming standard cheese: 4.7 (4.0-5.3) vs. 5.0 (4.3-5.5) mg/dL; (p: 0.056), while the predialysis serum total calcium did not differ: 9.3 (8.7-9.5) vs. $9.1(8.7-9.5) \mathrm{mg} / \mathrm{dL}(\mathrm{p}=0.820)$ between the 2 periods. Notably, there was no difference in the $\Delta \mathrm{P}$ observed between the run-in period and the period in which the standard cheese was consumed. Moreover, the subgroup of teenage patients did not experience different efficacy and safety compared with the adult patients: the inter-dialysis $\Delta \mathrm{P}$ values in teenagers consuming standard cheese and FriP cheese were $2.9(2.2-3.4) \mathrm{mg} / \mathrm{dL}$ and $2.8(1.8-2.9) \mathrm{mg} / \mathrm{dL}$, respectively, while the values in adults were $2.7(2.2-3.3)$ and 2.4 (1.9-2.7) $\mathrm{mg} / \mathrm{dL}$, respectively, and the pre-dialysis serum calcium levels in teenagers consuming standard cheese and 
Table 1 General characteristics of enrolled patients and the laboratory parameters at baseline and at the end of each study period

\begin{tabular}{|c|c|c|c|}
\hline \multicolumn{4}{|l|}{ Clinical and laboratory data } \\
\hline N. of patients & & & 16 \\
\hline Gender, M/F & & & $8 / 8$ \\
\hline Age, years & & & $26.9(14.9-69.0)$ \\
\hline Weight, $\mathrm{kg}$ & & & $58(51-69)$ \\
\hline \multirow[t]{2}{*}{ BMI $\left(\mathrm{kg} / \mathrm{m}^{2}\right)$} & & & $22.3(18.7-25.3)$ \\
\hline & Baseline & Standard cheese & FriP cheese \\
\hline Systolic BP & $124(112-135)$ & $124(112-135)$ & $129(123-140)$ \\
\hline Diastolic BP & $70(63-74)$ & $65(58-78)$ & $69(65-73)$ \\
\hline sPhosphorus, mg/dl & $4.9(4.1-5.7)$ & $5.0(4.3-5.5)$ & $4.7(4.0-5.3)$ \\
\hline sCalcium, mg/dl & $9.1(8.8-9.8)$ & $9.1(8.7-9.5)$ & $9.3(8.7-9.5)$ \\
\hline Ionized Calcium mmol/l & $1.23(1.15-1.25)$ & $1.21(1.19-1.26)$ & $1.23(1.21-1.23)$ \\
\hline PTH, ng/l & $96(61-144)$ & $92(54-214)$ & $81(45-185)$ \\
\hline ALP U/1 & $115(64-203)$ & $107(66-179)$ & $105(75-193)$ \\
\hline Cholesterol, mg/dl & $135(111-148)$ & $129(122-146)$ & $132(115-184)$ \\
\hline Triglycerides, mg/dl & $128(89-156)$ & $111(89-153)$ & $120(72-154)$ \\
\hline sBicarbonates, $\mathrm{mEq} / \mathrm{l}$ & $22.3(20.6-24.3)$ & $22.9(22.0-23.5)$ & $23.9(20.9-24.8)$ \\
\hline sCreatinine, mg/dl & $6.6(5.7-8.0)$ & $7.3(6-8.9)$ & $7.3(5.9-10.3)$ \\
\hline urea, $\mathrm{mg} / \mathrm{dl}$ & $128(120-165)$ & $153(103-184)$ & $139(111-181)$ \\
\hline Albumine, $\mathrm{g} / \mathrm{dl}$ & $4.1(3.9-4.3)$ & $4.2(4.0-4.5)$ & $4.2(3.9-4.5)$ \\
\hline
\end{tabular}

$s$ serum; $B M I$ body mass index, $A L P$ alkaline phosphatase, $B P$ blood pressure

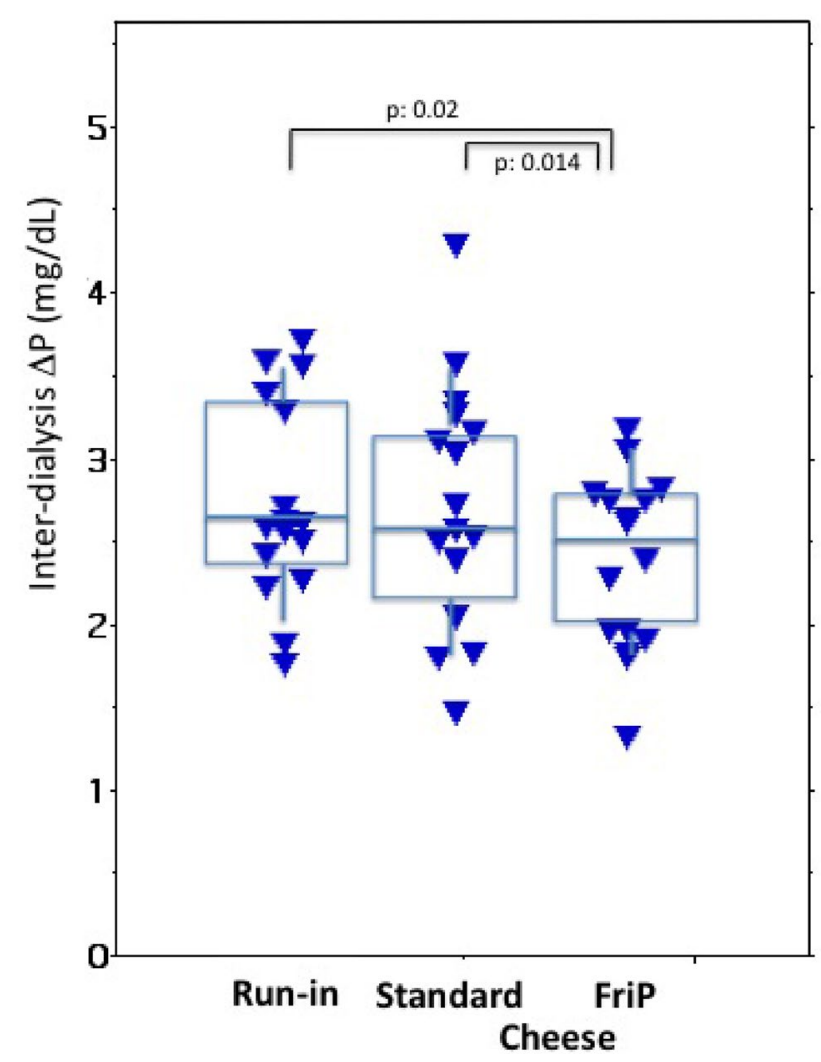

Fig. 1 Inter-dialysis increase in phosphorus levels in 16 patients on kidney replacement therapy during the run-in period (usual diet) and when exposed to standard cheese and FriP cheese
FriP cheese were 9.3 (9.1-10.1) and 9.4 (9.3-9.8), respectively, while the values in adults were 8.8 (8.5-9.1) and 8.7 (8.5-9.3) $\mathrm{mg} / \mathrm{dL}$, respectively.

Finally, no significant differences were observed between the standard cheese and FriP cheese periods in the interdialysis increase in body weight: $1.25(0.33-2.62)$ vs 1.29 $(0.25-2.87) \mathrm{kg}$ or in any of the other investigated variables (Table 1). All patients enjoyed both products equally, and the mean amount per $\mathrm{kg}$ of BW consumed per week was not different: 5.93 (3.21-7.78) gr of standard cheese vs. 4.96 (2.89-7.26) gr of FriP cheese (p: 0.56). No significant difference was observed between teenage and adult patients with regard to the amount of cheese consumed. All patients reported significant appreciation and gratification achieved by the reintroduction of cheese into their diet.

\section{Discussion}

Worldwide, the number of people on KRT is estimated to be more than 1.4 million, with the incidence growing by approximately $8 \%$ annually [3]. These patients must control their intake of sodium and fluids to avoid severe hypertension and fluid overload. They must also reduce their intake of fruits and vegetables to prevent hyperkalemia. Finally, the amount of meat, fish, milk and cheese consumed has to be limited to avoid hyperphosphatemia, one of the main determinants of secondary hyperparathyroidism $[4,5]$. 


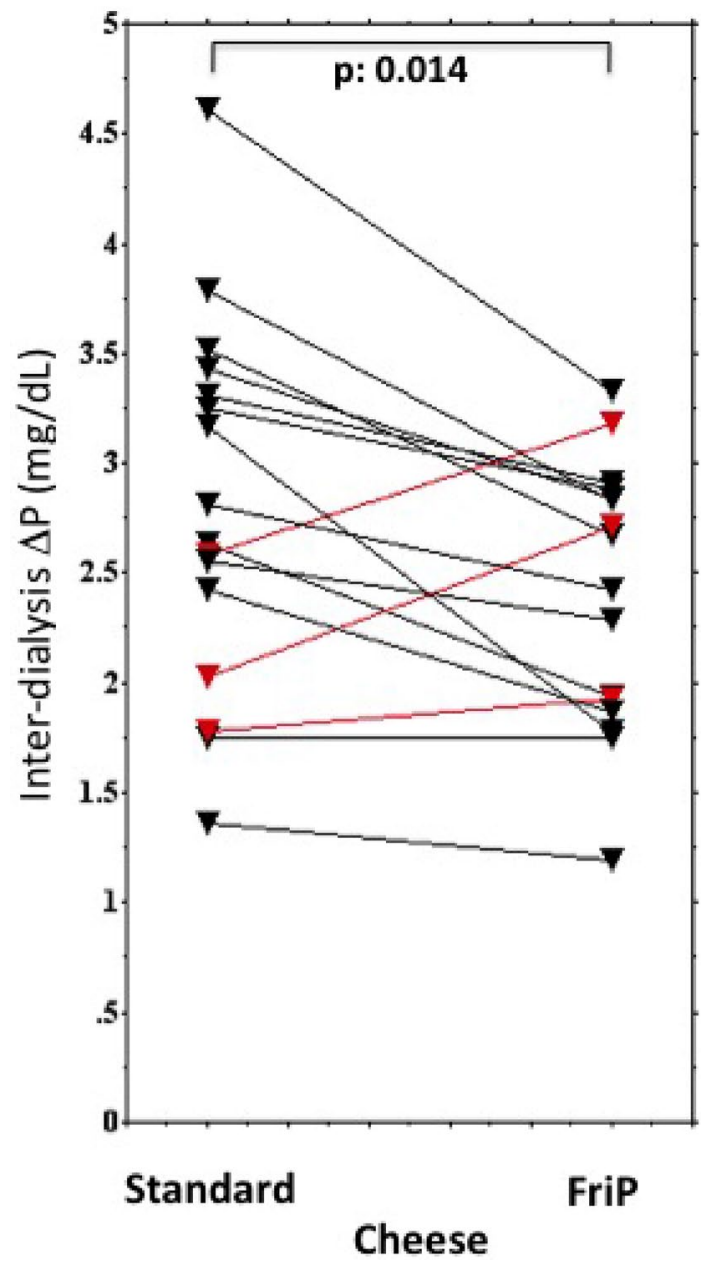

Fig. 2 Individual changes in inter-dialysis phosphorus increases in patients when exposed to standard cheese and to FriP cheese (the red lines indicate patients that experienced an increase in interdialysis phosphorus levels with FriP cheese)

Such extensive food restrictions ultimately lead to a reduction in food intake, malnutrition, depression and reduced compliance with both dietary and therapeutic prescriptions. This is especially true for people who are on maintenance dialysis for a long time [6,7].

The idea of producing a special kind of cheese, enriched with calcium carbonate, derived from our experience in the care of newborns with renal insufficiency and hyperphosphatemia. These patients cannot consume anything but milk, either breast milk or formula, both of which are characterized by a high content of phosphate. In the past, when phosphorus-free formulas were not available (as is currently the case with breast milk), the addition of calcium carbonate to the milk was the standard of care $[8,9]$. With FriP cheese, we simply applied the same concept to cheese-making, given the knowledge that calcium-enriched milk, in addition to trapping phosphorus, also favors the chemical process of caseification.
Moreover, since calcium carbonate is tasteless, the process of producing FriP cheese does not affect the taste of the final product and can be applied to any of the more than 2,000 different types of cheese available in Europe. We highlight that, according to the enrolled patients, the modified cheese was indistinguishable from the standard cheese and it was acknowledged to be an excellent product. The described process of producing FriP cheese offers the possibility to combine the best cheese-making tradition with the production of a product suitable for CKD patients (including a reduced content of sodium from average $1.2-1.5 \mathrm{gr} / 100 \mathrm{gr}$ of product to $<1 \mathrm{gr}$ ), providing patients with the opportunity to vary their diet, with positive effects on their phosphorus levels, diet and ultimately on their quality of life. The aim of the present study was exploratory, therefore, there are still several open issues, such as the long-term impact of added calcium on vascular calcifications and the impact of cheesederived caloric intake on body composition. However, we believe that the positive results of this preliminary evaluation support the feasibility of testing FriP cheese consumption in larger and longer studies that could address these unresolved issues.

A major limitation of the present pilot study, besides the small study sample, is that the patients' usual diet was not investigated or standardized. We expect that the cross-over design with random sequencing may have limited the potential bias. Moreover, we do not think that the patients' diets could be standardized for 3 months, and we do not feel very confident with regard to the reliability of dietary recall.

In conclusion, our study shows that $\mathrm{CaCO}_{3}$-enriched dairy products are efficacious, well-accepted and safe in patients on dialysis. Thus, we suggest their use in patients on dialysis to overcome some of their nutritional limitations and to increase their compliance to phosphate-binders, in order to achieve better phosphorous control and improved nutrition and quality of life.

\section{Declarations}

Conflict of interest The authors declare that they do not have any competing interests. FriP cheese is a trademark hold by Fondazione IRCCS Ca' Granda Ospedale Maggiore Policlinico.

Ethical statement The study was approved by the Ethical Committee.

\section{References}

1. Kurita N, Wakita T, Ishibashi Y, Fujimoto S, Yazawa M, Suzuki T, Koitabashi K, Yanagi M, Kawarazaki H, Green J, Fukuhara S, Shibagaki Y (2020) Association between health-related hope and adherence to prescribed treatment in CKD patients: multicenter cross-sectional study. BMC Nephrol 21(1):453. https:// 
doi.org/10.1186/s12882-020-02120-0 (PMID:33129292; PMCID:PMC7603681)

2. St-Jules DE, Rozga MR, Handu D, Carrero JJ (2020) Effect of phosphate-specific diet therapy on phosphate levels in adults undergoing maintenance hemodialysis: a systematic review and meta-analysis. Clin J Am Soc Nephrol 16(1):107-120. https://doi. org/10.2215/CJN.09360620 (PMID: 33380474)

3. Sarah LW, Steven JC, Stephen J, Jeremy RC, Alan C (2008) How can we achieve global equity in provision of renal replacement therapy? Bull World Health Organ. https://doi.org/10.2471/BLT. 07.041715

4. Nagasawa Y (2021) Positive and negative aspects of sodium intake in dialysis and non-dialysis CKD patients. Nutrients 13(3):951. https://doi.org/10.3390/nu13030951

5. Kalantar-Zadeh K, Tortorici AR, Chen JL, Kamgar M, Lau WL, Moradi H, Rhee CM, Streja E, Kovesdy CP (2015) Dietary restrictions in dialysis patients: is there anything left to eat? Semin Dial 28(2):159-168. https://doi.org/10.1111/sdi.12348 (PMID: 25649719; PMCID: PMC4385746)

6. National and Kidney Foundation (NKF) (2021) Potassium and your CKD diet. https://www.kidney.org/atoz/content/potassium.

\section{Authors and Affiliations}

Gianluigi Ardissino ${ }^{1} \cdot$ Roberto Matta $^{1} \cdot$ Mariagrazia Patricelli $^{1} \cdot$ Valentina Capone $^{1} \cdot$ Antonio Leoni $^{1}$. Antonio Groppelli ${ }^{2}$. Simone Vettoretti ${ }^{3}$. Laura Pavone ${ }^{3}$. Elisabetta Margiotta ${ }^{3}$. Annamaria Brancadoro ${ }^{3}$. Emanuele Grimaldi ${ }^{3} \cdot$ Isabella Cropanese ${ }^{4} \cdot$ Francesca Raffiotta $^{3} \cdot$ Piergiorgio Messa $^{3} \cdot$ Giovanni Montini $^{1}$

1 Pediatric Nephrology, Dialysis and Transplantation, Fondazione IRCCS Ca' Granda Ospedale Maggiore Policlinico, Milano, Italy

2 Cascina Rosina, Trucazzano, Italy

3 Nephrology and Dialysis, Fondazione IRCCS Ca' Granda Ospedale Maggiore Policlinico, Milano, Italy
7. Jeremy B, Ray F (1988) Factors influencing compliance with dietary restrictions in dialysis patients. J Psychosom Res 32(2):191196. https://doi.org/10.1016/0022-3999(88)90054-2 (ISSN 0022-3999)

8. Foster BJ, McCauley L, Mak RH (2012) Nutrition in infants and very young children with chronic kidney disease. Pediatr Nephrol 27(9):1427-1439. https://doi.org/10.1007/s00467-011-1983-x

9. Alon U, Davidai G, Bentur L, Berant M, Better OS (1986) Oral calcium carbonate as phosphate-binder in infants and children with chronic renal failure. Miner Electrolyte Metab 12(5-6):320325 (PMID: 3807830)

Publisher's Note Springer Nature remains neutral with regard to jurisdictional claims in published maps and institutional affiliations.

Accessed 15 Dec 2020 